Artigos Originais

\title{
Resultados de fertilização in vitro em mulheres submetidas previamente à laqueadura tubária
}

\author{
In vitro fertilization outcome for women previously submitted to tubal sterilization
}

Rosana Maria dos Reis ${ }^{1}$, George Dantas de Azevedo ${ }^{2}$, Paula Andrea de Albuquerque Salles Navarro ${ }^{3}$, Carlos Henrique Medeiros de Araújo ${ }^{4}$, Wellington de Paula Martins ${ }^{4}$, Rui Alberto Ferriani ${ }^{1}$, Marcos Felipe Silva de Sá ${ }^{1}$

\section{RESUMO}

Objetivos: avaliar os efeitos da laqueadura tubária sobre os resultados de fertilização in vitro com transferência de embrião. Métodos: estudo retrospectivo, no qual foram analisadas 98 mulheres submetidas a ciclos consecutivos de fertilização in vitro. Grupo LT, 55 mulheres com antecedente de laqueadura tubária bilateral, sem outros fatores de infertilidade associados, e grupo IM, 43 mulheres com infertilidade devido a fator masculino leve. As variáveis analisadas foram: idade, taxa de suspensão do ciclo de indução da ovulação, resposta à indução da ovulação (dias de indução, unidades de gonadotrofinas, número de folículos recrutados e oócitos captados), taxas de fertilização e de clivagem, número de embriões transferidos e gravidez clínica por ciclo de transferência. Resultados: não houve diferença entre as taxas de suspensão de ciclo de indução da ovulação, as respostas à indução da ovulação, as taxas de fertilização e de clivagem, o número de embriões transferidos e as taxas de gravidez clínica por ciclo transferido, entre os grupos LT e IM. Considerando-se isoladamente a variável idade no grupo LT, evidenciou-se que pacientes com idade superior a 35 anos utilizaram mais unidades de gonadotrofinas ( 2445 versus $2122 \mathrm{UI}$ ), tiveram menor número de folículos recrutados $(11,3$ versus 15,8$)$ e menos oócitos foram captados $(6,1$ versus 8,5$)$, quando comparadas às mulheres com idade de 34 anos ou menos. Conclusões: a laqueadura tubária não interferiu nos resultados da fertilização in vitro. Observamos uma piora da resposta à indução da ovulação nas pacientes submetidas à laqueadura tubária com idade superior a 35 anos.

PALAVRAS-CHAVE: Técnicas reprodutivas assistidas; Fertilização in vitro; Indução da ovulação; Laqueadura tubária; Transferência de embrião

\section{ABSTRACT}

Purpose: to investigate the effects of previous bilateral tubal sterilization on the outcome of in vitro fertilization. Methods: retrospective study of 98 consecutive in vitro fertilization cycles. Fifty-five women with previous tubal sterilization without any other infertility factor (TL group) were compared with 43 women with infertility due only to mild male factor (MI group. Age, cancellation rate per induction cycle, response to ovulation induction (number of days of ovulation induction, total amount of gonadotrophin units used, number of follicles and oocytes retrieved), fertilization and cleavage rates, number of transferred embryos and clinical pregnancy per transfer cycle were the variables considered. Results: the cycle discontinuation rate due to poor response, results of ovulation induction, fertilization and cleavage rates, number of transferred embryos and the occurrence of clinical pregnancy were similar in both groups. Considering solely the variable age in TL group, we observed that patients older than 35 years required higher gonadotrophin doses during ovulation induction (2445 versus 2122 IU), presented lower response with fewer follicular growth (11.3 versus 15.8) and less oocytes retrieved (6.1 versus 8.5) compared to younger women (34 years old or less). Conclusions: tubal sterilization did not interfere with in vitro fertilization outcomes. We observed a worse response to ovulation induction in women older than 35 years, who had previous tubal sterilization.

KEYWORDS: Reproductive techniques, assisted; In vitro fertilization; Ovulation induction; Sterilization, tubal; Embryo transfer

Setor de Reprodução Humana, Departamento de Ginecologia e Obstetrícia da Faculdade de Medicina de Ribeirão Preto da Universidade de São Paulo - USP - Ribeirão Preto (SP), Brasil.

1 Professor do Departamento de Ginecologia e Obstetrícia da Faculdade de Medicina de Ribeirão Preto da Universidade de São Paulo - USP - Ribeirão Preto (SP), Brasil.

2 Professor do Departamento de Morfologia da Faculdade Federal de Medicina da Universidade Federal do Rio Grande do Norte - UFNR - Rio Grande do Norte (RN), Brasil.

3 Médica Assistente do Departamento de Ginecologia e Obstetrícia do Hospital das Clínicas da Faculdade de Medicina de Ribeirão Preto da Universidade de São Paulo - USP - Ribeirão Preto (SP), Brasil.

4 Pós-Graduandos do Departamento de Ginecologia e Obstetrícia da Faculdade de Medicina da Universidade de São Paulo - USP - Ribeirão Preto (SP), Brasil.

Correspondência: Rosana Maria dos Reis

Departamento de Ginecologia e Obstetrícia da Faculdade de Medicina de Ribeirão Preto da Universidade de São Paulo

Avenida Bandeirantes, 3800 - 14049-900 - Ribeirão Preto - SP - e-mail: romareis@fmrp.usp.br

Recebido em: 2/8/2006

Aceito com modificações em: 6/11/2006

Rev Bras Ginecol Obstet. 2006; 28(12): 715-20. 
Introdução

A esterilização tubária é a técnica de contracepção definitiva realizada com maior freqüência no nosso meio. Estima-se que existam, no Brasil, mais de 5 milhões de mulheres em idade reprodutiva com antecedente de laqueadura tubária. Uma análise epidemiológica da situação mundial demonstra que a maioria das mulheres optou por esse método de contracepção definitiva numa idade inferior a 30 anos e em um período que apresentavam uma situação conjugal estável ${ }^{1}$.

Entretanto, um percentual significativo de mulheres arrepende-se após a realização da laqueadura tubária, sobretudo em virtude de mudanças em suas situações pessoais, sociais, financeiras e/ou em suas condições de saúde. Contudo, alguns autores demonstraram que a principal razão isolada que leva ao desejo de retorno à fertilidade é a mudança de parceiro e o anseio de ter um filho com o novo cônjuge ${ }^{2}$. Desta forma, a tranqüilidade que antes existia devido à contracepção definitiva agora dá lugar à infertilidade, que prevalece num grupo etário em que as opções de tratamento nem sempre oferecem resultados satisfatórios. Analisando o seguimento de 374 mulheres submetidas à esterilização tubária, ao longo de 16 anos, foi observado que $13,7 \%$ das pacientes arrependeram-se da decisão, $5,4 \%$ apresentavam-se insatisfeitas com a cirurgia e $6,5 \%$ desejavam a volta da fertilidade ${ }^{3}$.

No tratamento da infertilidade em mulheres anteriormente submetidas à laqueadura tubária duas opções estão disponíveis: a reanastomose tubária microcirúrgica e a fertilização in vitro e transferência de embriões.

A cirurgia de reanastomose tubária pode ser realizada pela via abdominal ou por abordagem laparoscópica ${ }^{4}$ e apresenta índices de sucesso na obtenção de gestação variando de 30 a 80\% Tomazevic e Ribic-Pucelj ${ }^{9}$ demonstraram uma taxa de gravidez cumulativa da ordem de $31 \%$, após cinco anos da cirurgia para o tratamento de patologias tubárias distais, ao passo que outros autores apontaram uma taxa cumulativa de gravidez de até $83,3 \%$, após 18 meses da cirurgia de reanastomose pós-esterilização tubária ${ }^{10}$.

As grandes discrepâncias entre os dados de literatura sobre a efetividade destes procedimentos cirúrgicos em possibilitarem a obtenção de gestação em mulheres previamente submetidas à laqueadura tubária devem-se principalmente a diferenças nas variáveis avaliadas, como as condições das trompas e a idade das pacientes analisadas. Os resultados da reanastomose tubária estão relacionados à integridade anatômica e funcional das trompas, sendo recomendada uma seleção adequada das pacientes candidatas ao método, uma vez que o sucesso da cirurgia depende essencialmente da existência prévia e atual de doença tubária ${ }^{11}$. Por outro lado, sabemos que a idade feminina avançada relaciona-se com a piora progressiva da fertilidade, devida, sobretudo, à redução da reserva folicular ovariana e ao comprometimento da qualidade oocitária, justificando as menores taxas de gestação em mulheres em idade reprodutiva avançada, quando comparadas a pacientes mais jovens ${ }^{12}$. Além dos resultados variáveis obtidos após a reanastomose tubária, a maior incidência de gravidez ectópica, descrita por alguns autores, constitui-se em outro aspecto relevante, que também deve ser levado em consideração quando da escolha pelo melhor procedimento a ser realizado para a obtenção de gestação neste grupo de mulheres ${ }^{13}$.

Alguns autores evidenciaram um aumento na resistência vascular ovariana, avaliada por ultra-sonografia com dopler pulsado, em pacientes submetidas à laqueadura tubária ${ }^{14}$. Desta forma, hipoteticamente, a laqueadura tubária poderia comprometer o fluxo sanguíneo ovariano e, conseqüentemente, promover uma piora da resposta à indução da ovulação e dos resultados da fertilização in vitro. Contudo, a maioria dos estudos publicados, acerca da influência da infertilidade tubária sobre os resultados da fertilização in vitro, refere-se a outras etiologias desta causa de infertilidade, principalmente as aderências tubárias e a hidrossalpinge ${ }^{15}$. Assim, não dispomos de dados conclusivos na literatura acerca dos potenciais efeitos da laqueadura tubária sobre a resposta à indução da ovulação e as taxas de gestação obtidas após a fertilização in vitro neste grupo de mulheres.

Atualmente, existe controvérsia acerca da decisão a ser tomada para uma paciente com antecedente de laqueadura tubária desejosa de gravidez. Se, por um lado, os resultados obtidos após as cirurgias de anastomose tubárias são bastante variáveis, por outro lado, a literatura é deficiente em informações acerca dos resultados de fertilização in vitro no grupo de pacientes laqueadas previamente. Desta maneira, o objetivo deste trabalho é avaliar os possiveis efeitos da laqueadura tubária sobre os resultados da fertilização in vitro, visando fornecer mais dados para auxiliar na decisão quanto ao melhor procedimento terapêutico a ser indicado para se tentar obter uma gestação neste grupo crescente de mulheres.

\section{Métodos}

Foi realizado um estudo retrospectivo de 98 mulheres submetidas a ciclos consecutivos de FIVETE, executados no Setor de Reprodução Humana do Hospital das Clínicas da Faculdade 
de Medicina de Ribeirão Preto da Universidade de São Paulo (USP).

As pacientes foram divididas em dois grupos: grupo LT ( $\mathrm{n}=55$ mulheres), constituido de mulheres com antecedente de laqueadura tubária bilateral, sem outros fatores de infertilidade associados, e grupo IM ( $\mathrm{n}=43$ mulheres), constituído de mulheres cujo fator responsável pela infertilidade era apenas o fator masculino leve. Foram incluídas no presente estudo apenas pacientes com idade entre 25 e 38 anos. Os critérios de exclusão foram: infertilidade masculina grave (concentração de espermatozóides móveis e progressivos no recuperado com valor inferior a $10.000 .000 / \mathrm{mL}$ ), ciclos realizados na mesma paciente, infertilidade por anovulação, obstrução tubária de outra origem (por exemplo, doença inflamatória pélvica) diagnosticada por hístero-salpingografia e endometriose.

Foram analisados 151 ciclos, e, destes, 21 foram excluídos por serem o segundo ciclo de paciente já incluída no estudo, 15 foram excluídas devido a endometriose, nove ciclos foram excluídos por se tratarem de infertilidade masculina grave e oito devido à anovulação.

Todas as pacientes foram submetidas à supressão hipofisária com agonista do hormônio liberador das gonadotrofinas (protocolo longo) previamente à indução da ovulação com gonadotrofinas exógenas. O crescimento folicular foi monitorizado por ultra-sonografias seriadas e a ovulação induzida com gonadotrofina coriônica humana (hCG) na dosagem de $10.000 \mathrm{UI}$, quando dois ou mais folículos atingiram um diâmetro médio superior a $18 \mathrm{~mm}$. A captação dos oócitos foi realizada 35 horas após a administração de hCG, por meio de punção guiada pela ultra-sonografia transvaginal. A transferência embrionária foi efetuada 48 a 72 horas após a captação oocitária. A fase lútea foi suplementada com progesterona micronizada associada ou não a injeções de hCG a cada três dias. O diagnóstico de gravidez clínica foi feito pela visualização ultra-sono- gráfica de saco gestacional quatro a cinco semanas após a transferência embrionária.

As variáveis analisadas foram: idade, resposta à indução da ovulação (representada pelo número de dias do ciclo de indução, unidades de gonadotrofinas utilizadas, número de folículos com diâmetro maior do que $12 \mathrm{~mm}$ por ocasião da administração de hCG e número de oócitos captados), taxas de fertilização e de clivagem embrionária, número de embriões transferidos e taxa de gestação clínica (número de gravidezes clinicamente diagnosticadas - exame ecográfico mostrando batimento cardíaco embrionário). Foram também avaliadas as taxas de suspensão do ciclo de indução da ovulação nos dois grupos, sendo estes casos excluídos da análise em relação aos resultados da transferência de embriões.

Os dados foram analisados através do programa GraphPad Prism ${ }^{\circledR}$, version 4.0, for Windows (GraphPad Software, San Diego, CA, USA). A normalidade da distribuição foi avaliada através do teste de normalidade D'Agostino and Pearson omnibus, sendo então calculadas as médias e desvios padrões. As médias foram comparadas através do teste $t$, pois todas as variáveis analisadas passaram pelo teste de normalidade, e as proporções foram avaliadas através do teste exato de Fisher. Foi considerado como diferença significativa o valor de $\mathrm{p}$ inferior a 0,05.

\section{Resultados}

Não houve diferença significativa quanto à idade das pacientes (grupo LT=33,5 $\pm 3,4$ anos e grupo $\mathrm{IM}=32,7 \pm 3,6$ anos) e quanto à taxa de suspensão de ciclos de indução da ovulação (grupo LT=dez casos ou $18,9 \%$ e grupo $\mathrm{IM}=$ oito casos ou $17,8 \%)$ entre os grupos avaliados.

Os resultados de indução da ovulação foram semelhantes nas pacientes dos dois grupos avaliados (Tabela 1). Não se observou diferença significativa entre as taxas de fertilização $(67,4$ versus $65,3 \%$,

Tabela 1 - Apresentação dos resultados da indução da ovulação e da transferência de embriões entre as mulheres com infertilidade devido à laqueadura tubária prévia (grupo LT) e devido a fator masculino leve (grupo IM).

\begin{tabular}{|c|c|c|c|}
\hline & Grupo LT (n=55) & Grupo IM (n=43) & $p$ \\
\hline Idade (anos) & $33,5 \pm 3,4$ & $32,7 \pm 3,6$ & $\mathrm{NS}^{\mathrm{a}}$ \\
\hline Folículos acima de $12 \mathrm{~mm}$ & $13,6 \pm 7,7$ & $12,4 \pm 5,6$ & $\mathrm{NS}^{\mathrm{a}}$ \\
\hline Oócitos captados & $7,7 \pm 4,3$ & $8,5 \pm 5,0$ & $\mathrm{NS}^{\mathrm{a}}$ \\
\hline Embriões transferidos & $2,7 \pm 1,3$ & $3,0 \pm 1,3$ & $\mathrm{NS}^{\mathrm{a}}$ \\
\hline Taxa de suspensão & $18,9 \%$ & $17,8 \%$ & $\mathrm{NS}^{b}$ \\
\hline Taxa de fertilização & $67,4 \%$ & $65,3 \%$ & $\mathrm{NS}^{\mathrm{b}}$ \\
\hline Taxa de clivagem & $84,6 \%$ & $86,4 \%$ & $\mathrm{NS}^{\mathrm{b}}$ \\
\hline Taxa de gravidez clínica & $24,3 \%$ & $27,4 \%$ & $N^{b}$ \\
\hline
\end{tabular}

Dados paramétricos apresentados como média \pm desvio padrão; NS=Diferença não significativa ( $p>0,05)$; a=dados comparados pelo teste $t$ de Student; $b=$ dados comparados pelo teste exato de Fisher. 
grupos LT e IM, respectivamente), taxas de clivagem embrionária (84,6 versus $86,4 \%$, grupos LT e IM) e na taxa de gravidez clínica $(24,3$ versus $27,4 \%$, grupos LT e IM) quando avaliadas pelo teste exato de Fisher. Também não houve diferença significativa na média do número de embriões transferidos entre os dois grupos $(2,7 \pm 1,3$ versus $3,0 \pm 1,3$, grupos LT e IM) quando analisados pelo teste $t$.

Em vista da ausência de diferença significativa entre os resultados obtidos pelos dois grupos estudados, foi realizada uma análise isolada das variáveis dentro do grupo de mulheres com laqueadura tubária prévia (grupo LT), em termos de resposta à indução da ovulação e taxa de gravidez. Os resultados obtidos nas pacientes com idade $\leq 34$ anos foram comparados àqueles referentes às mulheres na faixa etária de 35 anos ou mais (Tabela 2).

Observou-se um aumento significativo na dose de gonadotrofina utilizada e uma diminuição significativa do número de folículos recrutados e de oócitos captados nas pacientes com idade maior ou igual a 35 anos. Contudo, não se observou diferença significativa na taxas de fertilização e de clivagem embrionária e o número de embriões transferidos entre as duas faixas etárias avaliadas.

\section{Discussão}

Para a maioria das mulheres, a aceitação da esterilização tubária como método de controle da fertilidade tem sido baseada não somente na facilidade e na segurança dos procedimentos cirúrgicos envolvidos, mas também nas complicações e contra-indicações potenciais de outros métodos, como os contraceptivos orais e o dispositivo intrauterino. Entretanto, uma parcela significativa de mulheres se arrepende dessa decisão, procurando o ginecologista com o desejo de engravidar ${ }^{2,16}$.
Nessa situação, as duas opções possiviveis são a reanastomose tubária microcirúrgica e a fertilização in vitro com transferência de embriões, estando a decisão na dependência de diversos fatores, como idade da paciente, a concomitância com outros fatores de infertilidade, a possibilidade de acesso às técnicas de fertilização assistida, a capacidade técnica do médico em realizar a reanastomose cirúrgica das tubas uterinas, as condições anatômicas e funcionais dos cotos tubários, entre outros. O grande número de variáveis a serem analisadas, as diferenças entre as taxas de gestação descritas obtidas por meio destas duas opções terapêuticas e as potenciais complicações oriundas das mesmas fazem com que, atualmente, exista grande controvérsia acerca da decisão a ser tomada para uma paciente com antecedente de laqueadura tubária desejosa de gravidez.

A fertilização in vitro representa, na atualidade, uma terapêutica alternativa real e disponivel na maioria dos centros urbanos. No entanto, a literatura contém dados insuficientes acerca dos efeitos potenciais da laqueadura tubária sobre os resultados da transferência de embriões.

No presente estudo não foi evidenciada diferença entre as respostas à indução da ovulação, as taxas de fertilização e de clivagem, o número de embriões transferidos e as taxas de gravidez clinica por ciclo transferido, entre as pacientes previamente submetidas à laqueadura tubária e o grupo controle, representado por mulheres cujo fator responsável pela infertilidade era apenas o fator masculino leve. A análise conjunta destes dados sugere que a laqueadura tubária per se não interfere nos resultados da fertilização in vitro, elucidando uma dúvida até então pouco explorada na literatura. Nossos dados confirmam os obtidos por Sitko et al. ${ }^{17}$, que também não evidenciaram diferenças no número de embriões transferidos e nas taxas de gravidez obtidas através da

Tabela 2 - Distribuição dos resultados da indução da ovulação, fertilização in vitro e transferência de embriões nas mulheres com laqueadura tubária prévia, segundo o limite estabelecido de idade de 35 anos.

\begin{tabular}{|c|c|c|c|}
\hline & $25-34$ anos $(n=33)$ & $35-38$ anos $(n=22)$ & $p$ \\
\hline Idade (anos) & $30,3 \pm 2,5$ & $36,8 \pm 0,9$ & $<0,0001^{a}$ \\
\hline Dose de gonadotrofina (UI) & $2122 \pm 403$ & $2445 \pm 502$ & $0,01^{a}$ \\
\hline Dias de indução & $9,3 \pm 2,1$ & $9,4 \pm 1,9$ & $\mathrm{NS}^{\mathrm{a}}$ \\
\hline Folículos recrutados & $15,8 \pm 7,9$ & $11,2 \pm 7,2$ & $0,03^{\mathrm{a}}$ \\
\hline Oócitos captados & $8,5 \pm 3,3$ & $6,1 \pm 3,20$ & $0,001^{\mathrm{a}}$ \\
\hline Embriões transferidos & $3,15 \pm 1,23$ & $2,81 \pm 1,42$ & NS ${ }^{a}$ \\
\hline Taxa de fertilização & $70,7 \%$ & $63,4 \%$ & $N S^{b}$ \\
\hline Taxa de clivagem & $85,5 \%$ & $81,7 \%$ & $N S^{b}$ \\
\hline
\end{tabular}

Dados paramétricos apresentados como média \pm desvio padrão; NS=Diferença não significativa ( $p>0,05)$; a=dados comparados pelo teste $t$ de Student; $b=$ dados comparados pelo teste exato de Fisher.

Rev Bras Ginecol Obstet. 2006; 28(12): 715-20. 
transferência de embriões, quando compararam pacientes previamente submetidas à laqueadura tubária com mulheres portadoras de infertilidade secundária a patologias tubárias. Porém, convém destacar que o grupo controle do presente estudo, constituído por mulheres cujo fator responsável pela infertilidade era apenas o fator masculino leve, parece ser mais adequado para se avaliar a influência isolada da laqueadura tubária sobre os resultados da fertilização in vitro, pois exclui a influência potencial de outros fatores femininos de infertilidade. Outros autores, ao analisarem as influências de doenças tubo-ovarianas e cirurgias tubárias prévias sobre os resultados da fertilização in vitro, também não evidenciaram repercussões negativas de tais condições patológicas sobre os resultados destes procedimentos de reprodução assistida ${ }^{18}$, reforçando o achado de que a infertilidade de origem tubária não interfere negativamente.

Foi evidenciado que há maior utilização de gonadotrofinas e a obtenção de um menor número de oócitos captados em pacientes com laqueadura tubária prévia, submetidas à estimulação ovariana controlada, com idade superior a $38 \operatorname{anos}^{17}$. Contudo, no presente estudo, evidenciou-se que as pacientes previamente submetidas à laqueadura tubária com idade superior a 35 anos já apresentaram pior resposta à indução da ovulação do que as pacientes mais jovens, demonstrando que mulheres mais velhas apresentam menores probabilidades de obtenção de oócitos e implantação embrionária ${ }^{19}$. Apesar de a idade ser um fator que sabidamente afeta negativamente os resultados de tratamentos de infertilidade ${ }^{20}$, o achado do presente estudo define um ponto de corte etário a partir do qual as pacientes previamente submetidas à laqueadura tubária apresentariam piores resultados na fertilização in vitro.

Desta forma, concluímos que a laqueadura tubária não interfere nos resultados da fertilização in vitro e transferência de embriões e que as mulheres previamente submetidas à laqueadura tubária com idade superior a 35 anos apresentam pior resposta à indução da ovulação do que as mulheres mais jovens. Assim, se por um lado, a laqueadura tubária não interfere negativamente nos resultados da fertilização in vitro, por outro lado, a maioria dos estudos sugere que as cirurgias de reanastomose tubária microcirúrgica apresentam um bom prognóstico, principalmente em mulheres jovens ${ }^{4,21}$. Desta maneira, acreditamos que ambos os procedimentos devam ser considerados mais como complementares do que como competitivos, sendo a adequada seleção das pacientes, mediante a análise conjunta das diversas variáveis acima mencionadas, o ponto crucial para se determinar a melhor abordagem terapêutica em cada caso individual.

\section{Agradecimentos}

À equipe do Laboratório de Reprodução Humana: Maria Cristina Picinato M. de Araújo, Marilda H. Yamada Dantas, Maria Aparecida Carneiro Vasconcelos, Maria Albina Verceze Bortolieiro, Sandra A. Cavichiollo Viana, Maria Auxiliadora P. Rosa, Ilza Alves Rezende Mazzocato, Luiz Alberto Maneta e Stael Porto Leite.

\section{Referências}

1. Osis MJ, Faundes A, de Sousa MH, Duarte GA, Bailey P. Fertility and reproductive history of sterilized and non-sterilized women in Campinas, Sao Paulo, Brazil. Cad Saúde Pública. 2003;19(5):1399-404.

2. Machado KM, Ludermir AB, da Costa AM. Changes in family structure and regret following tubal sterilization. Cad Saúde Pública. 2005;21(6):1768-77.

3. Korell M, Englmaier R, Hepp H. [Effects of tubal sterilization[. Zentralbl Gynakol. 2000;122(1):28-34. German.

4. Gomel V, McComb PF. Microsurgery for tubal infertility. J Reprod Med. 2006;51(3):177-84.

5. Kim SH, Shin CJ, Kim JG, Moon SY, Lee JY, Chang YS. Microsurgical reversal of tubal sterilization: a report on 1,118 cases. Fertil Steril. 1997;68(5):865-70.

6. Goldberg JM, Falcone T. Laparoscopic microsurgical tubal anastomosis with and without robotic assistance. Hum Reprod. 2003;18(1):145-7.

7. Ribeiro SC, Tormena RA, Giribela CG, Izzo CR, Santos NC, Pinotti JA. Laparoscopic tubal anastomosis. Int J Gynaecol Obstet. 2004;84(2):142-6.

8. Wiegerinck MA, Roukema M, van Kessel PH, Mol BW. Sutureless re-anastomosis by laparoscopy versus microsurgical re-anastomosis by laparotomy for sterilization reversal: a matched cohort study. Hum Reprod. 2005;20(8):2355-8.

9. Tomazevic T, Ribic-Pucelj M. Microsurgery and in vitro fertilization/embryo transfer for infertility resulting from distal tubal lesions. J Reprod Med. 1991;36(7):527-30.

10.Yoon TK, Sung HR, Kang HG, Cha SH, Lee CN, Cha KY. Laparoscopic tubal anastomosis: fertility outcome in 202 cases. Fertil Steril. 1999;72(6):1121-6.

11.Posaci C, Camus M, Osmanagaoglu K, Devroey P. Tubal surgery in the era of assisted reproductive technology: clinical options. Hum Reprod. 1999;14 Suppl 1:120-36. 
12.Kailas NA, Sifakis S, Koumantakis E. Contraception during perimenopause. Eur J Contracept Reprod Health Care. 2005;10(1):19-25.

13.Barjot PJ, Marie G, Von Theobald P. Laparoscopic tubal anastomosis and reversal of sterilization. Hum Reprod. 1999;14(5):1222-5.

14. Revel A, Abramov Y, Yagel S, Nadjari M. Utero-ovarian morphology and blood flow after tubal ligation by the Pomeroy technique. Contraception. 2004;69(2):151-6.

15. Van Voorhis BJ. Outcomes from assisted reproductive technology. Obstet Gynecol. 2006;107(1):183-200.

16. Moseman CP, Robinson RD, Bates GW Jr, Propst AM. Identifying women who will request sterilization reversal in a military population. Contraception. 2006;73(5):512-5.

17.Sitko D, Commenges-Ducos M, Roland P, Papaxanthos-Roche A, Horovitz J, Dallay D. IVF following impossible or failed surgical reversal of tubal sterilization. Hum Reprod. 2001;16(4):683-5.

18. Oehninger S, Scott R, Muasher SJ, Acosta AA, Jones HW Jr, Rosenwaks Z. Effects of the severity of tubo-ovarian disease and previous tubal surgery on the results of in vitro fertilization and embryo transfer. Fertil Steril. 1989;51(1):126-30.

19. Qublan HS, Malkawi HY, Tahat YA, Areidah S, Nusair $\mathrm{B}$, Khreisat BM, et al. In-vitro fertilisation treatment: factors affecting its results and outcome. J Obstet Gynaecol. 2005;25(7):689-93.

20.Addy DM, Whitman-Elia GF, Queenan JT Jr. Age and resting follicle number predict response to gonadotropin stimulation in in vitro fertilization cycles. Am J Obstet Gynecol. 2002;187(2):285-7.

21. Granberg M, Strandell A, Thorburn J, Daya S, Wikland M. Economic evaluation of infertility treatment for tubal disease. J Assist Reprod Genet. 2003;20(8):301-8. 